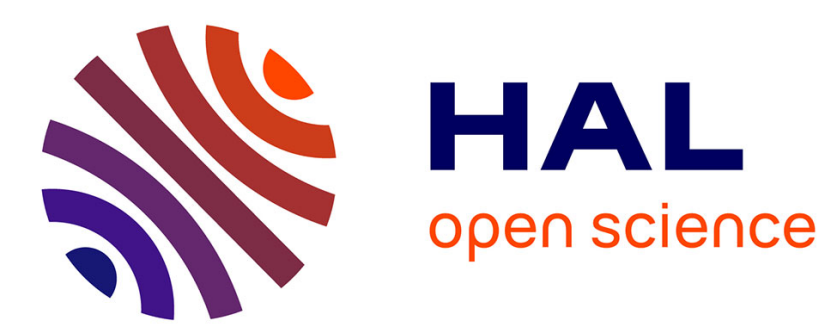

\title{
Undulation instability in stripe domain structures of " bubble " material
}

D. Sornette

\section{To cite this version:}

D. Sornette. Undulation instability in stripe domain structures of " bubble " material. Journal de Physique, 1987, 48 (1), pp.151-163. 10.1051/jphys:01987004801015100 . jpa-00210417

\section{HAL Id: jpa-00210417 https://hal.science/jpa-00210417}

Submitted on 1 Jan 1987

HAL is a multi-disciplinary open access archive for the deposit and dissemination of scientific research documents, whether they are published or not. The documents may come from teaching and research institutions in France or abroad, or from public or private research centers.
L'archive ouverte pluridisciplinaire HAL, est destinée au dépôt et à la diffusion de documents scientifiques de niveau recherche, publiés ou non, émanant des établissements d'enseignement et de recherche français ou étrangers, des laboratoires publics ou privés. 
Classification

Physics Abstracts

$75.70 \mathrm{~K}-75.60 \mathrm{E}-64.70 \mathrm{M}$

\title{
Undulation instability in stripe domain structures of « bubble » material
}

\author{
D. Sornette $(*)$ \\ Laboratoire de Physique de la Matière Condensée (CNRS UA 190) Université des Sciences, Parc Valrose,
} 06034 Nice Cedex, and, Collège de France, 11 place Marcelin Berthelot 75231 Paris Cedex 05, France

(Reçu le 19 juin 1986, révisé le 22 septembre, accepté le 24 septembre 1986)

\begin{abstract}
Résumé. - Des motifs bi-dimensionnels en lamelles apparaissent spontanément dans les films de matériau magnétique " à bulles ». Nous développons une analogie en terme de cristaux liquides smectiques pour expliquer les hystérésis topologiques observées récemment en appliquant des cycles de champs magnétiques. Nous montrons en particulier qu'un tel système en ruban subit une instabilité d'ondulation quand la valeur maximale du champ magnétique du cycle augmente. Nous décrivons tour à tour (1) l'instabilité d'ondulation analogue à celle des smectiques, (2) sa transformation en une structure en chevrons, (3) l'apparition de " digitations " à l'origine d'une structure finale très complexe en « labyrinthe ». Une comparaison quantitative est faite avec les données existantes.

Abstract. - Two-dimensional smectic-like patterns form spontaneously in garnet plates of bubble material. We use the smectic analogy to explain the origin of the topological hysteresis of these stripe domain structures recently observed under magnetic field cycles. We show that these systems undergo an undulation instability as the magnetic field peak increases. We describe successively (1) the undulation instability similar to that of smectics A, (2) its transformation into a chevron structure and (3) the formation of fingers resulting in a complex labyrinth structure. Quantitative comparison is made with existing experiments.
\end{abstract}

\section{Introduction.}

A topological hysteresis in stripe domain structures of bubble materials has recently been observed [1] by applying magnetic field cycles. The sample was a single crystal layer of formula

$$
\mathrm{Y}_{2,77} \mathrm{Bi}_{0,25} \mathrm{~Pb}_{0,02} \mathrm{Fe}_{3,99} \mathrm{Ga}_{1,06} \mathrm{Pt}_{0,01} \mathrm{O}_{12}
$$

grown on a Gadolinium Gallium Garnet substrate along the (111) direction (The typical dimension of the plate is. $\left.5 \times 10^{3} \times 5 \times 10^{5} \times 5 \mu \mathrm{m}\right)$. Previous studies [2] have used other materials having different quantitative characteristics but without qualitative change of the main behaviour.

Among the wide variety of stable and metastable domain structures [3], the stripe ones are reproducible and sufficiently stable since they are separated from the cylindrical-domain phase by energy barriers which cannot be overcome at room temperature in a zero magnetic field. These structures consist of alternate vertical up and down magnetization domains of respective width $d_{+(-)}$(see Fig. 1).

$\left(^{*}\right)$ Also at Centre de Physique théorique, Ecole Polytechnique. F-91128 Palaiseau, France.

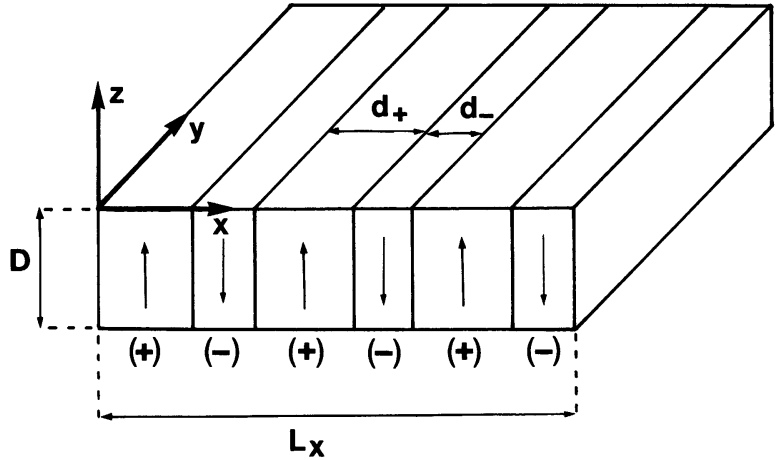

Fig. 1. - Schematic representation of the layers structure.

This stripe structure results from a compromise between demagnetization and the energy of Bloch walls. The material is sufficiently anisotropic and thin (thickness $D \sim 7 \mu \mathrm{m}$ ) so that no closure domains appear in the direction perpendicular to the sample [5].

The observations of reference [1] are summarized in figure 2. The sample is subjected to a magnetic cycle $\mathrm{O} \rightarrow H_{\max } \rightarrow \mathrm{O}$ of maximum magnetic field $H_{\max }$. Depending on $H_{\text {max }}$, different regimes occur. As soon as $H$ is non-zero, $d_{+}(-)$increases (decreases). The ques- 


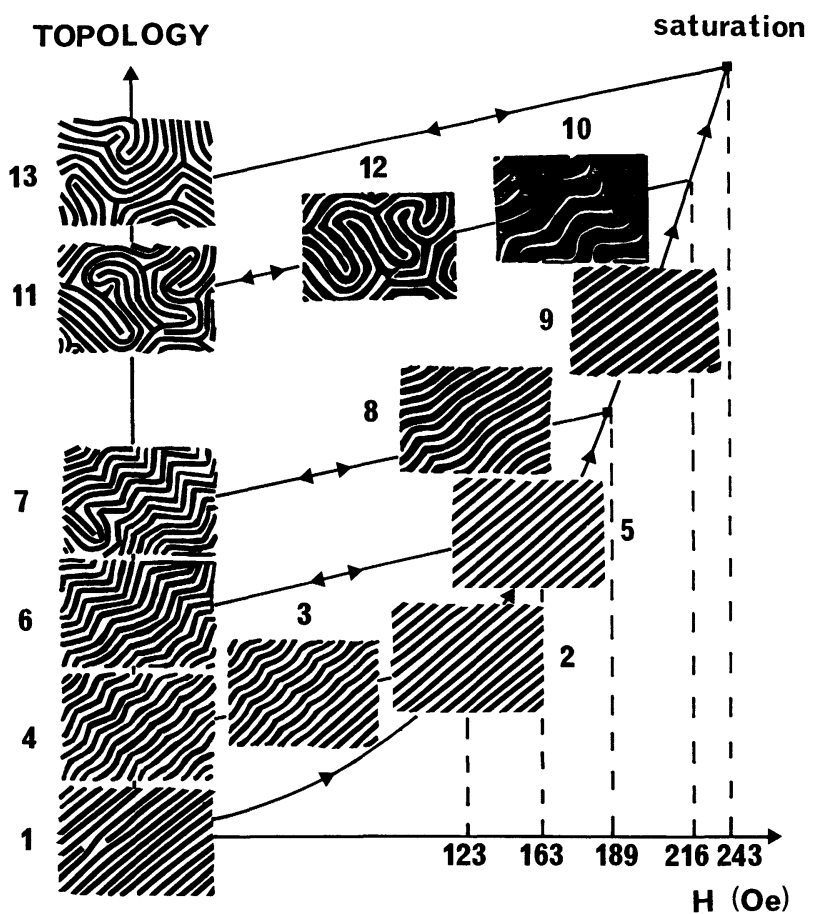

Fig. 2. - « Topology/Magnetic field » phase diagram (taken from Ref. [16] with permission) showing the topological hysteresis cycles of the stripe structure.

tion is : does the structure return to its initial topology when $H$ is decreased back to zero. For $H_{\max }<H_{1}$, the answer is yes : the process is reversible and the parallel stripe structure with the initial width $d_{+(-)}$is recovered. For $H_{\max }>H_{1}$, the structure finally obtained consists of undulating parallel layers. As $H_{\max }$ increases, the amplitude of the undulation increases and finally for $H_{\max }>H_{2}$, the undulated domains transform into a chevron structure. At still higher fields $\mathrm{H}_{\max }>\mathrm{H}_{3}$, fingers (i.e. single layers of determined magnetization) grow from the spikes of the chevron structure and result in a complex labyrinth topology.

In this work, we propose an explanation of these observations [1], based on a smectic analogy [4]. The main message of this paper is that many of the experimental observations made on magnetic stripe domain structures can be rationalized by local energy considerations. Indeed, it is possible to develop a fruitful analogy with smectic liquid crystals and to forget almost everything about long-range dipolar forces, exchange interactions..., once local elastic contributions such as bending and compression energies are defined. In other words, a new language and a new way of reasoning are proposed for understanding the deformations and distorsions in stripe magnetic structures.

The main feature of the system under consideration is the rotational invariance of the Hamiltonian controlling the layer deformations. This rotational invariance is very general in one-dimensional systems and occurs for example in Rayleigh-Bénard rolls. In this case, the Hamiltonian formulation exposed below is replaced by the Hohenberg-Swift phase equation which also describes an undulation instability of the rolls (the socalled « zig-zag » pattern) [9]. The undulation instability observed in magnetic films is therefore much more general and will occur as the signature for a breaking of rotational invariance.

The paper is organized as follows. We first recall the microscopic ingredients at the basis of the phenomenological model used. The analysis of Garel and Doniach [4] based on a Landau-Ginzburg expansion is first recalled in section 2 . This method allows us to derive the expression of the Hamiltonian describing distorsions of the stripe structure, which is found to have a smectic A form.

The effect of a magnetic field can be described within this theory but is useful only in the small field linear regime $\left(d_{+}-d_{-} \sim H\right)$. At higher fields, strong nonlinear behaviours are observed and adequately explained using the classical magnetostatic potential formalism [2]. Progressive extractions of stripe domain dislocations (SDD) is shown to occur as $H$ increases. This process is controlled by several forces such as the magnetically induced Peach-Koehler forces exerted on these SDD, the forces on dislocation cores exerted by the magnetic field and the defects, and the "elastic" interactions between SDD, as discussed in section 3 . After « one » or several walls have been excluded and the magnetic field is decreased back to zero, the period $d$ of the stripe structure is equal to that under field, which is larger than the initial period $d_{0}=d_{+}+d_{-}$in zero field. The «lamellar» structure is no longer at a local minimum of the energy and the system can be viewed as subjected to an extensional strain.

In section 4 , we analyse the consequences of the existence of this effective extensional strain and show that the stripe structure undergoes an undulation instability similar to that observed in smectic A liquid crystals. We predict the amplitude and wavelength of the modulation of the stripes and compare them with experimental observations. Semi-quantitative estimates of the energy of different configurations are given to explain the successive topologies observed as one goes to higher $H_{\max }$.

Section 5 concludes by raising some unresolved questions.

\section{Smectic form of the stripe structure energy.}

2.1 Heuristic APPROACH. - Consider a slab with a strongly uniaxial (Ising-like) material (see Fig. 1). At 《low » temperatures and zero applied field, the system breaks up into domains due to the competition between exchange and dipolar forces, with a zero net magnetization. The physical reason is that, due to its thickness and its strong magnetic anisotropy along $z$ (typical values are $k_{1} \sim-600 \mathrm{erg} / \mathrm{cm}^{3}, \quad k_{\mathrm{u}} \sim$ 
$5000 \mathrm{erg} / \mathrm{cm}^{3}, 4 \pi M_{\mathrm{s}} \sim 230$ Oe where $k_{1}$ and $k_{\mathrm{u}}$ are respectively the anisotropy constant in the plane and along $z$, and $M_{\mathrm{s}}$ is the saturation magnetization), closure domains do not exist (no formation of closure domains in the $z$ direction in order to close the magnetic field lines inside the sample [5]). For small $D$, the system can only develop domain walls parallel to the $z$ axis resulting in an important magnetic energy. By multiplying the domains (decreasing the period $d$ ), the system creates a field of high multipolar order whose range and strength and therefore magnetic energy decrease with $d$. This behaviour competes with the energy necessary for creating walls. We can thus write the energy of the system per unit surface as [2] :

$$
E \sim\left[r D / d+\alpha(d) \mu_{0} M_{\mathrm{s}}^{2} d\right]
$$

where $\alpha(d)=\sum_{n \text { odd }} 1 / n^{3} \cdot\left(1-\mathrm{e}^{-n \pi D / d}\right)$ is obtained by the sum over the mute index $n$. It is a slowly varying function of $d$ for $\pi D / d$ larger than unity. $r$ is the domain wall surface tension, $M_{\mathrm{s}}$ the saturation magnetization and $\mu_{0}$ the magnetic permeability. The first term represents the walls energy and the second term is the demagnetization contribution. Minimization of $E$ with respect to $d$ yields an equilibrium distance $d$ scaling with $D^{1 / 2}$. This treatment is allowed if $2<D / \xi<20$ [3] where $\xi$ is the width of the Bloch walls and if «branching » structures are neglected (see Ref. [5] for details on the branching phenomena).

The nucleation of the walls and the resulting patterns depend strongly upon the thermal and magnetic history of the sample. Note that a good way for obtaining a sample almost free of defects is to cool the sample under zero magnetic field from a temperature above the critical temperature (as done in Ref. [1]).

Reference [2] examined different magnetic histories and showed in particular the existence of nucleation centres from which star-like patterns of dislocations build up. In the following, we do not consider these cases but study almost perfect structures as obtained in reference [1].

2.2 LANDAU-GINZBURG THEORY. - Garel and Doniach have developed an alternative theory based on a Landau-Ginzburg approach which yields essentially the same qualitative results [4] as equation (1). In this paragraph, we just transcribe their results. They write the total free energy as the sum of two contributions, the exchange energy $\int \mathrm{d} S F_{\text {exch }}$ and the magnetic dipolar energy $\int \mathrm{d} S F_{\mathrm{D}}$ :

$$
F_{\mathrm{S}}=\int \mathrm{d} S\left(F_{\text {exch }}+F_{\mathrm{D}}\right)
$$

where $\mathrm{d} S$ is the differential surface element. The exchange energy is given by

$$
\begin{aligned}
F_{\text {exch }}=(D / 2 a) & J(\nabla m)^{2}+ \\
+ & \left(D / 2 a^{3}\right)\left(T-T_{\mathrm{F}}\right) m^{2} \\
& +D T_{\mathrm{F}} m^{4} / 12 a^{3}-g \mu_{\mathrm{B}} H m D
\end{aligned}
$$

where $m(x)$ is the slab average magnetization along $z, J$ the nearest-neighbour ferromagnetic exchange, $g$ the gyromagnetic coefficient, $\mu_{\mathrm{B}}$ the Bohr magneton per unit volume of the material and $a$ the lattice spacing. $T_{\mathrm{F}}$ is the mean field ferromagnetic transition temperature $T_{\mathrm{F}}=z J$ where $z$ is the coordination number.

The dipolar magnetic energy can be expressed as

$$
F_{\mathrm{D}}=1 / 2 \int \mathrm{d} S \sigma \phi
$$

where $\sigma=\mathbf{m} \cdot \mathbf{n}$ is the charge distribution of the surface created by the magnetization $\mathbf{m}, \mathbf{n}$ is the normal to the surface plate and $\phi$ is the magnetic potential on the surface created by $\sigma . F_{\mathrm{D}}$ is calculated in reference [2].

In the samples studied in reference [1], anisotropy exists but is much smaller than these two contributions. For the moment, we neglect it. We will come back to its role in breaking the rotational degeneracy.

The magnetic profile $m$ which minimizes the total free energy must be searched in a large space of functions dependent on position since it is easy to verify that $m=$ Const. is not a minimum. The simplest ansatz is given by the following parametrization

$$
m=m_{0}+m_{\mathrm{s}} \cos q_{0} x
$$

which corresponds to a truncation to the first term in a mode expansion. $m_{0}$ is the constant background of the magnetization whereas $m_{\mathrm{s}}$ is the amplitude of its spatial modulation whose period is $2 \pi / q_{0}$. The value of $m_{0}$ is obtained by minimization of

$$
f_{1}=1 / 2 f_{0} m_{0}^{2}+1 / 4 \cdot u m_{0}^{4}-h m_{0}
$$

with

$$
f_{0}=k\left(T-T_{0}\right) D / 2 a^{3}, T_{0}=T_{\mathrm{F}}-8 \pi\left(g \mu_{\mathrm{B}}\right)^{2} a^{3}
$$

is a shifted transition temperature, $u=k T_{\mathrm{F}} D / 3 a^{3}$ and $h=H D g \mu_{\mathrm{B}} \cdot f_{0}, u$ and $h$ have the dimension of an energy per unit surface. Expression (6) is nothing more than a Landau expansion in powers of the average magnetization $m_{0}$. In particular, $m_{0}$ is non-zero under zero magnetic field only when $f_{0}$ becomes negative i.e. when $T<T_{0}$.

The amplitude $m_{\mathrm{s}}$ is obtained from the minimization of

$$
f_{2}=\left(1 / 4 f_{q_{0}}+3 / 4 u m_{0}^{2}\right) m_{\mathrm{s}}^{2}+3 / 32 u m_{\mathrm{s}}^{4}
$$


where

$$
f_{q}=\left(T-T_{\mathrm{F}}\right) D / 2 a+q^{2} J D / a+4 \pi\left(g \mu_{\mathrm{B}}\right)^{2} / q
$$

$q_{0}$ is obtained by minimization of $f_{q}: \delta f_{q} / \delta q=0$. This gives

$$
q_{0}=\left(2 \pi a\left(g \mu_{\mathrm{B}}\right)^{2} / J D\right)^{1 / 3} .
$$

Note that $q_{0}$ is independent of the temperature as it should in the rather « low » temperature regime where one can neglect deviation of the magnetization (far from the walls) from the saturation magnetization. Near the critical temperature, relevant deformation energies become small so that thermal fluctuations become important. This regime has been studied in reference [11].

Equation (9) shows that the period $d$ scales as $D^{1 / 3}$ and is independent of the applied magnetic field when $h$ is small enough so that the single mode approximation (5) remains valid.

This is in contradiction with the scaling $d \sim D^{1 / 2}$ deduced from equation (1). The difference stems from the fact that in equation (1), the Bloch walls are assumed of negligible thickness resulting in a magnetization profile which is flat on intervals of width $d$ (groove-like profile which includes all harmonic orders). On the other hand, equation (9) relies on the first harmonic approximation (5) : it yields an energy proportional to $q \sim d^{-1}$ per period and therefore an energy per unit surface scaling as $q^{2} \sim d^{-2}$ as seen in the second term of the r.h.s. of expression (8). This comparison shows the limitation of the Landau approximation: the underlying assumption is that higher harmonics grow more slowly at temperatures just below the phase transition $T_{\mathrm{F}}$. The present analysis holds only in a limited temperature range such that $T$ is low enough $\left(T_{\mathrm{F}}-T>1 \mathrm{~K}\right)$ for $M$ not to depend crucially on $d$ and $T$ is not too far from $T_{\mathrm{F}}$ such that only a small number of harmonics appear.
Apparently, this remark casts strong doubts on the validity of the theory developed below for describing instabilities of stripes obeying a $D^{1 / 2}$ law. However, the main assumption underlying the analysis is the phase approximation used under a particular form in equation (11), which consists in developing $m(x)$ under the form $m(x)=m_{\mathrm{s}} \Pi\{x-u(x \cdot y)\}$ where $\Pi(x)$ can be any periodic function such as the groove profile assumed in equation (1). From this phase approximation, one can obtain quite generally a Hamiltonian in terms of increasing powers of spatial derivatives of the layer displacement $u$ and recover the smectic free energy (17) from the lowest orders.

Let us come back to the Landau-Ginzburg free energy. The key point stressed in reference [4] is that in this Landau-Ginzburg formalism, the competition between the wall energy $\left((\nabla m)^{2}\right.$ term $)$ and the dipolar term yields a non-zero optimal wave vector as in equation (1) ; the direction of this wave vector is arbitrary in the $x-y$ plane if the isotropy of the system is preserved. Note, however, that this orientational invariance can be broken by the presence of boundary effects, by defects or by an anisotropic energy $k_{1} \neq 0$. In the sample studied in reference [1], $k_{1}$ is not strictly zero $\left(\sim-600 \mathrm{erg} / \mathrm{cm}^{3}\right)$ and introduces a symmetry of order three in the plane $(x, y)$. This is felt at high magnetic field cycles for which the effective curvature rigidity modulus $K$ becomes very small (see the top of Fig. 2 where the symmetry of order three can be guessed).

Taking $\mu_{\mathrm{B}} /$ mole $=7 \times 10^{-6}$ MKSC, a density of 5 and a molar mass of $\sim 30 \mathrm{~g}$ yields a typical magnetization of $7 \times 10^{-6} .5 \times 10^{3} / 0.03 \sim 1$ MKSA. Using $J \sim k T_{\mathrm{F}} / z$ with $T_{\mathrm{F}}=400 \mathrm{~K}$ (Ref. [1]) and the coordination number $z=6$, the gyromagnetic factor $g=2$, the lattice spacing $a \sim 12.83 \AA, D=7 \mu \mathrm{m}$ yields from equation (9) : $d=2 \pi / q_{0} \sim 3 \mu \mathrm{m}$, which is the right order of magnitude (Ref. [1] measures $d=5 \mu \mathrm{m}$ ).

Away from this minimum (9), the perturbations are described by a Hamiltonian which in the harmonic approximation reads [4] :

$$
F_{\mathrm{s}} \sim\left(J D / a q_{0}^{2}\right) \int \mathrm{d}^{2} x\left[\left(\nabla^{2} m\right)^{2}-2 q_{0}^{2}(\nabla m)^{2}+q_{0}^{4} m^{2}-a q_{0}^{2} h m / J D\right]
$$

2.3 SMECTIC-LIKE FREE ENERGY. - Let us introduce the phase approximation,

$$
m(x)=m_{\mathrm{s}} \cos [q\{x-u(x, y)\}]
$$

where $u(x, y)$ describes, in a continuous framework, the slowly varying positive displacement along $x$ of the portion at position $(x, y)$ of the wall at position $x$ (we note $x-u(x, y)$ in the cos so that $u(x, y)$ describes the displacement counted as positive along the $x$ direction). Inserting (11) in (10), we obtain (in zero magnetic field) :

$$
\begin{aligned}
F_{\mathrm{s}} \sim\left(J D / a q_{0}^{2}\right) m_{\mathrm{s}}^{2} \int \mathrm{d}^{2} x\left\{\left(q^{2}-q_{0}^{2}\right)^{2}\right. & +2\left(q^{2}-q_{0}^{2}\right)\left[-2 q^{2} \partial u / \partial x+(\nabla u)^{2}\right]+ \\
& \left.+4 q^{4}(\partial u / \partial x)^{2}+q^{2}\left(\nabla^{2} u\right)^{2}-4 q^{2}(\partial u / \partial x)(\nabla u)^{2}+q^{4}(\nabla u)^{4}\right\}
\end{aligned}
$$


This Hamiltonian has been considered in reference [6] to obtain the effect of the first non-harmonic corrections on the renormalization by thermal fluctuations of the curvature and compression moduli defined below.

To obtain $\langle\partial u / \partial x\rangle=0$, one must choose $q=q_{0}$.

To quadratic order in $u$, expression (12) then reduces to a smectic A liquid crystal Hamiltonian :

$$
F_{\mathrm{s}}=\int \mathrm{d}^{2} x\left[1 / 2 B(\partial u / \partial x)^{2}+1 / 2 K\left(\partial^{2} u / \partial y^{2}\right)^{2}\right]
$$

where the compression and curvature moduli are respectively

$$
\begin{aligned}
& B=8 J D m_{\mathrm{s}}^{2} q_{0}^{2} / a \\
& K=2 J D m_{\mathrm{s}}^{2} / a
\end{aligned}
$$

$\lambda=\sqrt{K / B}$ is the so-called penetration length equal, in our problem, to $1 / 2 q_{0}^{-1}=d / 4 \pi$.

Note the interesting result that the deformation energy of the stripe structure does not involve a surface tension term proportional to $(\partial u / \partial y)^{2}$ if the condition $q=q_{0}$ is satisfied. This is the well-known consequence of the spatial isotropy of the lamellar structures (the small in-plane anisotropy is neglected here).

Under zero magnetic field, $m_{s}^{2}=2 / 3\left(-f_{q_{0}}\right) / u$. Let us write $f_{q_{0}}=D\left(T-T_{q_{0}}\right) / 2 a^{3}$ as in reference [4] with $T_{q_{0}} \sim T_{\mathrm{F}}-8 \pi\left(g \mu_{\mathrm{B}}\right)^{2} a^{3} / q_{0} D$ which is greater than $T_{0}$. With the numerical values taken above, we have $T_{\mathrm{F}}-T_{0} \sim 300 \mathrm{~K}$ and $T_{\mathrm{F}}-T_{q_{0}} \sim 30 \mathrm{~K}$. For the range of temperatures $T_{0}<T<T_{q_{0}}$,

$$
m_{0}=0
$$

and

$$
m_{\mathrm{s}}^{2}(h=0) \quad\left(T_{q_{0}}-T\right) / T_{\mathrm{F}}
$$

where we have used $u \sim k T_{\mathrm{F}} D / a^{3}$. Reporting expression (15) in equation (14) yields

$$
K \sim 8 J\left\{\left(T_{q_{0}}-T\right) / T_{\mathrm{F}}\right\} D / a
$$

which for $d=7 \mu \mathrm{m}, a=2 \AA, T_{q_{0}}-T \sim 10 \mathrm{~K}, T_{\mathrm{F}}=$ $400 \mathrm{~K}$ yields $K \sim 10^{3} k T_{\mathrm{F}}$. The proportionality of $K$ on $D / a$ was expected on physical grounds. Such a high value for the curvature rigidity and therefore for the compression moduli explains why thermal fluctuations are completely negligible, as observed experimentally $[1,2]$. In particular, the discussion of Garel and Doniach, on the possible observation of unbound pairs of dislocations and of the corresponding absence of long-range order, does not seem relevant to these experimental samples.

For the sequel where we study instabilities, it is important to remark that, as for smectic liquid crystals, expression (13) is incomplete since it is not invariant under global rotation of the sample as it should due to the arbitrary in the direction of the optimal wave vector $q_{0}$. The argument follows the smectic one [7] and is illustrated in figure 3. A given rotation $\theta$ of a perfectly aligned stripe with spacing $d$ yields an effective distance between layers, counted in the $x$ direction, given by $d_{\text {cff }}=d / \cos \theta$. The differential displacement along $x$, $\partial u / \partial x$, away from the true equilibrium spacing $d$ is therefore

$$
(d / \cos \theta-d) 1 / d \sim \theta^{2} / 2=1 / 2(\partial u / \partial y)^{2}
$$

for small $\theta$.

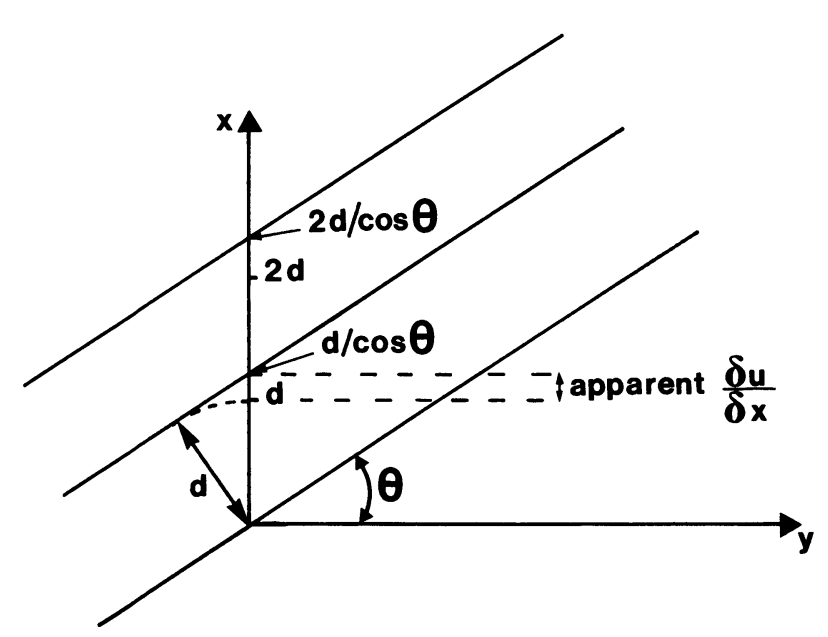

Fig. 3. - Tilted layers. Representation of the apparent extension.

\section{Consequently, a $x$-deformation}

$$
\partial u / \partial x \sim 1 / 2(\partial u / \partial y)^{2}
$$

corresponds to a pure rotation and does not involve additional energy. A true $x$-deformation is therefore $\partial u / \partial x-1 / 2(\partial u / \partial y)^{2}$ and expression (13) must be modified and reads up to third order in $u$ :

$$
\begin{aligned}
F_{\mathrm{s}}=\int \mathrm{d}^{2} x\{1 / 2 B[\partial u / \partial x & \left.-1 / 2(\partial u / \partial y)^{2}\right]^{2}+ \\
+ & \left.1 / 2 K\left(\partial^{2} u / \partial y^{2}\right)^{2}\right\} .
\end{aligned}
$$

This is precisely the form obtained directly in equation (12) for $q=q_{0}$ within the phase approximation (11). However, the preceding argument shows, in addition to the phase approximation, that other terms of higher order in $u$ (coming from the expansion of $\cos \theta$ ) must be included. This will be discussed in section 4.1 .

This type of Hamiltonian is well known in the context of smectic A elasticity and is responsible for the undulation instability occurring under extensional strain [7]. Below, we shall show that a similar behaviour appears under magnetic field cycles. 


\section{Effect of a magnetic field.}

3.1 EFFECT OF A MAGNETIC FIELD ON THE STRIPE DOMAINS.

3.1.1 Linear regime. - For the range of temperatures $T_{0}<T<T_{q_{0}}, m_{0}$ is zero under $h=0$ and increases linearly with $h$. From equation (6), we obtain

$$
m_{0} \# h / f_{0} \# 2 a^{3} h / D\left(T-T_{0}\right)
$$

which has a paramagnetic form. In order to relate $m_{0}$ to the change of thickness of $(+)$ and $(-)$ domains, note that one can define the new position of the $(+) /(-)$ walls by setting $m\left(x_{\mathrm{w}}\right)=0$ :

$$
m\left(x_{\mathrm{w}}\right)=m_{0}+m_{\mathrm{s}} \cos \left(2 \pi x_{\mathrm{w}} / d\right)=0 .
$$

For small $h$ (i.e. small $m_{0}$ ), this yields the thickness of the $(+)$ and $(-)$ domains :

$$
\begin{aligned}
& d_{+}=2 x_{\mathrm{w}}=d / 2\left(1+2 m_{0} / \pi m_{\mathrm{s}}\right) \\
& d_{-}=d-d_{+} .
\end{aligned}
$$

Equation (6) can be translated in terms of the variable $d_{+}(-)^{-} d / 2$, and we thus obtain the leading term in the free energy describing a deviation of $d_{+}\left(d_{-}\right)$ from $d / 2$ :

$$
\begin{array}{r}
f_{1}=\left(\pi^{2} / 8\right) f_{0} m_{\mathrm{s}}^{2}\left\{2\left(d_{+}(-)^{-d / 2}\right) / d\right\}^{2}- \\
-\pi h m_{\mathrm{s}}\left(d_{+(-)}-d / 2\right) / d .
\end{array}
$$

We have neglected, at this order, the change of $m_{\mathrm{s}}$ due to the non-vanishing $m_{0}$ (cf. Eq. (7)). Expression (21) allows us to obtain the pressure exerted on a wall when $d_{+}(-) \neq d / 2$. It reads

$$
\begin{aligned}
& p_{+(-)}= \\
&=\left(-\pi^{2} / 4\right) f_{0} m_{\mathrm{s}}^{2}\left(d_{+(-)}-d / 2\right) /(d / 2)^{2}- \\
&-\pi h m_{\mathrm{s}} / d .
\end{aligned}
$$

In the absence of defects, $d_{+}\left(_{-}\right)$adjusts itself so that $p=0$ which yields the corresponding relative width increment of $(+)$ domains :

$$
\begin{aligned}
2\left(d_{ \pm}-d / 2\right) / d= \pm 8 & \left(g \mu_{\mathrm{B}}\right) a^{3} / \pi\left(T-T_{0}\right) \times \\
& \times\left\{T_{\mathrm{F}} /\left(T_{q_{0}}-T\right)\right\} H
\end{aligned}
$$

which scales with $H$. This gives typically $2\left(d_{+}-d / 2\right) / d \sim 10^{-3}-10^{-4} H$ (with $H$ in oersted). This compares well with the data from reference [1] where an applied field of $50 \mathrm{Oe}$ results in a $2\left(d_{+}-d / 2\right) / d$ of a few percent.

Note that the magnetic field has also the effect of decreasing the curvature rigidity modulus $K$ defined in equation (14). This stems from the dependence of $m_{\mathrm{s}}$ on $h$ via the $3 u m_{0}^{2} / 4$ factor in the second term of the r.h.s. equation (7) :

$m_{\mathrm{s}}^{2}=32\left\{\left(-f_{q_{0}}\right) / 3 u\right\}\left\{1-3 u m_{0}^{2} / 4\left(-f_{q_{0}}\right)\right\}$.

The corresponding reduction of $K$ is therefore quadratic in the applied field.

3.1.2 Non linear regime. - The linear regime and the symmetry $d_{+}-d / 2=-\left(d_{-}-d / 2\right)$ described by equation (23) breaks down for high magnetic fields $(H>\sim 50$ Oe [1] ). This can be fully analysed within the classical magnetostatic potential formalism followed in reference [2] and outlined in equation (1) in the limit of complete saturation at the centre of each layer.

Equation (1) is the special case $H=0$ implying $M=0 \quad$ (with $M / M_{\mathrm{s}}=\left(d_{+}-d_{-}\right) /\left(d_{+}+d_{-}\right), M_{\mathrm{s}}$ being the saturation magnetization). The general case $H \neq 0$ has been completely solved in reference [2]. It accurately takes into account the magnetic long range interactions between domains. This is in contrast with the LG expansion (2) and (3) yielding equations (6) and (7) where only local correlations are described.

The results of the analysis of reference [2] are summarized in figure 4 , which shows the dependence of $d_{+}(H), d_{-}(H)$ and the period $d(H)=$ $d_{+}(H)+d_{-}(H)$ on $H$. In particular it shows that $d(H)$ remains approximately constant for $H / 4 \pi M_{\mathrm{s}}>\sim 0.2(H<\sim 50 \mathrm{Oe})$ and increases rapidly for higher fields. This stems from the fact that the width $d_{+}$increases very rapidly with increasing field, whereas the width $d_{-}$decreases only slightly. For $d_{+}$to increase more rapidly than $d_{-}$decreases, some of

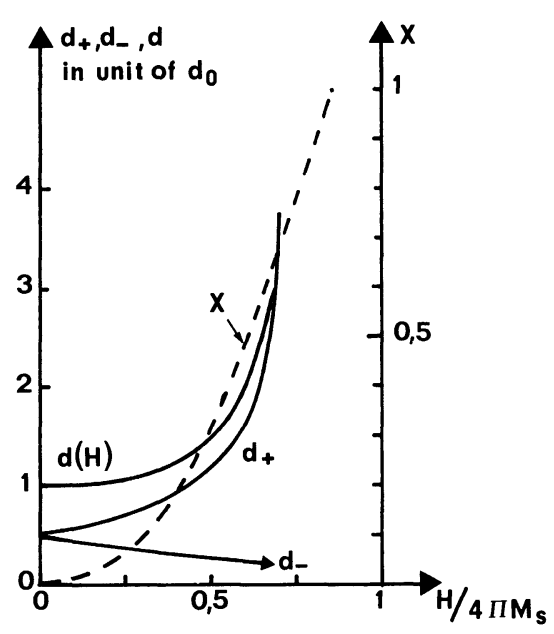

Fig. 4. - Variations of the thicknesses $d_{+}\left\{d_{-}\right\}$of $(+)\{(-)\}$ domains, the period $d(H)=d_{+}+d_{-}$and the strain $X=\left(d(H)-d_{0}\right) / d(H)$ as a function of $H$ (taken from Ref. [2]). For comparison with reference [1], $4 \pi M_{\mathrm{s}}=266$ oersted and $d_{0}=5 \mu \mathrm{m}$. 
the (- ) domains must disappear. However, experiments show that those $(-)$ domains which disappear do not disappear by reducing their width to zero but by diminishing their length $[1,2]$. We now explain how this can occur.

3.2 EXClusion OF Dislocations : ROLE OF PEACHKOEHLER FORCES [8]. - Let us consider the stripe structure of initial period $d_{0}(H=0)$ submitted to a magnetic field $H$. Since the equilibrium period is $d(H)>d_{0}$, the layers are under compression. This results in a Peach-Koehler force exerted by this strain on dislocations. It reflects the tendency for the system to relax towards the equilibrium spacing by motions of dislocations. We note the compression of the sample $\delta=N\left(d-d_{0}\right)$ where $N=L_{x} / d_{0}$ is the initial number of layers. $L_{x}$ is the width of the plate defined in figure 1.

Let us consider a single dislocation of Burgers vector $b$ contained on the left of the $x-y$ plane and with its core at $y_{0}$. We assume that the elastic distortion energy of the "smectic» structure is still described by the Hamiltonian (13). At equilibrium, the compression energies per unit surface $L_{x} L_{y}$ are [7] :

$$
\begin{array}{ll}
E_{1}=1 / 2 B\left\{(-\delta-b / 2) / L_{x}\right\}^{2} L_{x} y_{0} & \text { in region } y<y_{0} \\
E_{2}=1 / 2 B\left\{(-\delta+b / 2) / L_{x}\right\}^{2} L_{x}\left(L_{y}-y_{0}\right) & \text { in region } y>y_{0} .
\end{array}
$$

The total energy is therefore :

$$
E=E_{1}+E_{2}=C-B \delta b y_{0} / L_{x} .
$$

$C$ is a constant independent of $y_{0}$ taking into account of the curvature energy associated with the distortion of the $(+)$ and $(-)$ layers in the vicinity of the dislocation core. With $\delta=N\left(d-d_{0}\right)$, we obtain the force on the dislocations along $y$ :

$$
f_{\mathrm{PK}}=\delta E / \delta y_{0}=-B b\left(d-d_{0}\right) / d_{0} .
$$

This force $\left(f_{\mathrm{PK}} \sim 10^{-11}\left(d-d_{0}\right) / d_{0}\right.$ newtons $)$ tends to extract the dislocations and is proportional to the compressional strain $\left(d-d_{0}\right) / d_{0}$. This seems to be the explanation for the observation that the $(-)$ domains do not disappear by reducing their width to zero but by diminishing their length [1].

Note that other forces on the dislocations are present, coming from the elastic interaction between dislocations and the action of the magnetic field and the defects on the dislocation core. They also seem to be important quantitatively and they are discussed in the appendix.

\section{Topological instabilities of the stripe structure.}

Let us summarize the physical picture obtained so far.

The free energy describing the distortion of the stripe structure has been found to have a smectic-like form. As a consequence, one expects to recover, in the magnetic stripe problem, many features characteristic of smectic liquid crystals. Indeed, in the following, we develop the consequences of expression (17) in the presence of strains induced by magnetic fields and find that the topological deformations found in these systems have their smectic counterparts.

In the presence of (SDD) dislocations, a magnetic field has the effect of extruding some of them such as to obtain the optimal period under field $d(H)$. Upon decreasing the field, the period remains equal to $d(H)$ due to the irreversibility of the nucleation of domains [1-3]. At the end of a field cycle $0 \rightarrow H \rightarrow 0$, the stripe structure is under extensional strain $\delta=N\left(d(H)-d_{0}\right)$ with $N(H)=L_{x} / d(H)$. The system may choose to relax the strain by tilting the layers locally (see the discussion of $\S 2.3$ leading to expression (17) of the Hamiltonian) as we now discuss.

4.1 UNDULATION INSTABILITY. - The treatment closely follows that developed for smectics $[7,8]$ and we include some details for completeness. The physical meaning of the undulation instability comes from the competition between the extensional energy given to the system by the magnetic field cycle and the opposing elastic bending energy which is the response to the system for resisting any external constraint.

We start from the Hamiltonian (17). In the presence of a uniform external strain $X=\delta / L_{x}=$ $\left(d(H)-d_{0}\right) / d_{0}$, it transforms into :

$$
F_{\mathrm{s}}=\int \mathrm{d}^{2} x\left[1 / 2 B\left(X+\partial u / \partial x-1 / 2(\partial u / \partial y)^{2}\right)^{2}+1 / 2 K\left(\partial^{2} u / \partial y^{2}\right)^{2}\right]
$$

To obtain (28), we have reparametrized $u$ under the form $u_{\text {new }}=u_{\text {old }}-X x$, so that $u_{\text {new }}$ can be chosen periodic in $x$. In the following, we omit the index and note $u=u_{\text {new }}$.
The extremum condition for $F_{\mathrm{s}}$ gives the Euler equation :

$$
\left(\partial^{2} u / \partial x^{2}-X \partial^{2} u / \partial y^{2}\right) / \lambda^{2}-\partial^{4} u / \partial y^{4}=0 .
$$


Let us first analyse the effect of a localized perturbation : $u=u_{0} \sin k y$ at $x=0$. We look for solutions in the form $u=u_{0} \mathrm{e}^{\sigma x} \sin k y$. Inserting this form in equation (29) yields :

$$
\sigma=-k\left(\lambda^{2} k^{2}-X\right)^{1 / 2} .
$$

We have taken the - sign since it is the analytical continuation of the exponentially relaxing solution for $X=0$. Equation (30) shows that when $X>\lambda^{2} k^{2}, \sigma$ becomes purely imaginary. This means that the localized perturbation propagates throughout the sample. However, at $X=\lambda^{2} k^{2}, \sigma=0$ which implies free boundary conditions $\left(u \neq 0\right.$ at $\left.x= \pm L_{x} / 2\right)$. In reality, the boundary conditions impose $u\left( \pm L_{x} / 2, y\right)=0$. This amounts to pose :

$$
u=u_{0} \cos \left(\pi x / L_{x}\right) \sin (k y)
$$

where $L_{x}$ is the width of the sample containing $L_{x} / d_{0}$ initial stripes. This is equivalent to expanding the solution on the set of eigenfunctions of the linear operator $\mathbb{L}$. (Eq. (29) can indeed be written $\mathbb{L} u=0$.) This writes that the distortion is periodic along $x$ for the first mode of interest (for the higher modes : $u=$ $\left.u_{0} \cos \left(n \pi x / L_{x}\right) \sin (k)\right)$. Inserting (31) in (29), we obtain :

$$
X(k)=\lambda^{2}\left(k^{2}+k_{\mathrm{c}}^{4} / k^{2}\right)
$$

where $k_{\mathrm{c}}^{2}=\pi / \lambda L_{x}$ which corresponds to an undulation period $\Lambda=\left(d L_{x}\right)^{1 / 2}$. Equation (32) has no solution for $X$ less than the strain threshold $X_{\mathrm{c}}=X\left(k_{\mathrm{c}}\right)=$ $2 \lambda^{2} k_{\mathrm{c}}^{2}$. Using $\lambda=d / 4 \pi$ (Eq. (14)), it corresponds to a dilation $\delta_{0}=2 \pi \lambda=d_{0} / 2$ independent of $L_{x}$. Using $\delta=L_{x}\left(d(H)-d_{0}\right) / d(H)$, this yields $d(H)=$ $d_{0}\left(1-d_{0} / L_{x}\right)^{-1} \# d_{0}\left(1+d_{0} / L_{x}\right)$. From the curve $d(H)$ in figure 4 , this determines $H_{1} \cdot H_{1}$ is not universal since it depends on $L_{x}$. Using $L_{x} \sim 10^{3} \mu \mathrm{m}$, $d_{0} \sim 5 \mu \mathrm{m}$ yields $H_{1} \sim 50 \mathrm{Oe}$.

Around $X_{\mathrm{c}}, X(q)$ has the usual parabolic form of the marginal stability curve of a control parameter as a function of wave vectors. For $X<X_{\mathrm{c}}$, the stripe structure remains flat : the extensional energy given to the system by the magnetic field cycle is not large enough for compensating the cost in curvature energy associated with an undulation deformation. At $X=X_{\mathrm{c}}$, the marginal mode $k=k_{\mathrm{c}}$ becomes unstable. This occurs as soon as a single dislocation is extruded (which corresponds to $\delta=d$ ).

In order to predict the amplitude of the deformation above, but near, threshold, we must use a higher order form of $F_{\mathrm{s}}$ :

$$
\begin{aligned}
& F_{\mathrm{s}}=\int \mathrm{d}^{2} x F \\
& F_{\mathrm{s}}=\int \mathrm{d}^{2} x\left[(K / \dot{2}) \lambda^{2}\left(X+\partial u / \partial x-1 / 2 \theta^{2}+1 / 24 \theta^{4}\right)^{2}+1 / 2 K^{2} \theta^{2}\right]
\end{aligned}
$$

where $\theta=\partial u / \partial y$ and $X=\delta / L_{x}$. We have included the $\left\{\theta^{4}\right\}$ term coming from the expansion of cos $\theta$ (see $\S 2.3$ ). Note that to be consistent, we should have included all terms up to third powers in $u$ in equation (33). In particular, $1 / 2 K\left(\partial^{2} u / \partial y^{2}\right)^{2}$ should be replaced by

$$
1 / 2 K\left\{\partial^{2} u / \partial y^{2}(1+\delta u / \delta x)-\left(\delta^{2} u / \delta x \delta y\right)(\delta u / \delta y)\right\}^{2}
$$

based on the rotational invariance arguments [8]. However, these corrections involve amplitudes proportional to $1 / L_{x}$ which are small compared to $k_{0}$.

Using again the parametrization (31), we compute the free energy integrated over the surface $L_{x} \times 1$ and obtain it as a polynomial in $u_{0}^{2}$ (taking the integration over $y$ on a unit interval) :

$1 / L_{x} \int_{0}^{1} \mathrm{~d} y / k \int_{-L_{x} / 2}^{+L_{x} / 2} \mathrm{~d} x F / K=4 X^{2} / \lambda^{2}-\left(\pi k^{2} / \lambda^{2}\right)(X-X(k)) u_{0}^{2}+1 / 2\left(\left(9 k^{4} / 16\right) \lambda^{2}+\pi^{4} k^{2} / L_{X}^{4}\right) u_{0}^{4}$

where $X(k)$ is given by equation (32). Equation (34) is similar to a Landau expansion in the amplitude $u_{0}(k)$. For $X<X(k), u_{0}=0$ is the solution which minimizes $F_{\mathrm{s}}$. For $X>X(k)$, we recover the result that $u_{0}(k)$ becomes non-zero. This occurs for the first time at $k=k_{\mathrm{c}}\left(X=X_{\mathrm{c}}\right)$. For $X>X_{\mathrm{c}}$, all modes $k$ such that $X>X(k)$ have an energy $F_{\mathrm{s}}$ lower than the flat configuration $u_{0}=0$. This case pertains to the class of problems involving interactions between patterns (modes) [9]. Since $L_{y}$ is large, this is a « large box wave number selection problem» [10]. However, in our case, the problem simplifies since a variational proced- 
ure exists (minimization of the free energy). The optimal wave vector and its amplitude are given by the two extremal conditions :

$$
\delta F / \delta k^{2}=0 ; \quad \delta F / \delta u_{0}^{2}=0 .
$$

This yields

$$
\begin{gathered}
k_{\mathrm{extr}}-k_{\mathrm{c}} \sim \lambda\left(X-X_{\mathrm{c}}\right) / L_{x}^{3} k_{\mathrm{c}} \\
u_{0}=4 / 3 \times\left\{\lambda L_{x}\left(X-X_{\mathrm{c}}\right)\right\}^{1 / 2}= \\
=4 / 3 \times\left\{\lambda L_{x}\left(d(H)-d_{0}\right) / d(H)\right\}^{1 / 2} .
\end{gathered}
$$

Equation (35) shows that the wavelength of the ondulation decreases inversely proportionally to $X-X_{\mathrm{c}}$. The energy corresponding to solution (35) is :

$$
\begin{aligned}
F_{\mathrm{s}} & \sim K\left\{4 X^{2} / \lambda^{2}-8 k^{2} L_{x}\left(X-X_{\mathrm{c}}\right)^{2} / 9 \lambda\right\} \\
& \sim 4 K\left\{X^{2}-2 \pi^{2}\left(X-X_{\mathrm{c}}\right)^{2} / 9\right\} / \lambda^{2} .
\end{aligned}
$$

The negative second term of the r.h.s. is the energy gained by the system going from the flat to the undulation state.

The condition for the validity of the present theory is $\theta \ll 1$ i.e. $u_{0} \ll\left(\lambda L_{x} / \pi\right)^{1 / 2}$ i.e. $X-X_{\mathrm{c}} \ll 1$ or $\left(d(H)-d_{0}\right) / d(H) \ll 1$.

Taking typically $L_{x} \sim 1 \mathrm{~cm}, d=5 \mu \mathrm{m}, \delta=d$ yields $X \sim 05, \quad X_{\mathrm{c}} \sim 03, \quad \Lambda=2 \pi / k_{\mathrm{c}} \# 22 \mu \mathrm{m} \sim 4 d_{0} \quad$ and $u_{0} \sim 0.27 d \sim 1.3 \mu \mathrm{m}$. This order of magnitude is compatible with reported observations [1]. A systematic experimental study of the dependence of $k_{\mathrm{c}}$ (i.e. $\Lambda$ ) on $L_{x}$ and of $u_{0}$ on $H$ would be interesting for comparison with these predictions.

4.2 BEYOND THE UNDULATION INSTABILITY. - For higher values of the maximum applied field, the corresponding strain $\delta$ becomes much larger than $\delta_{c}$ and strong nonlinear behaviours leading to complicated patterns are expected. By analogy with smectic liquid crystals, one expects that different observations such as Grandjean terraces near boundaries, instabilities of edge dislocations, focal conics, walls, dislocations walls, etc. can have their counterparts in the 2D magnetic stripe structures. Let us consider some of them.

4.2.1 Discontinuity and curvature walls [8, 12]. Layer configurations as shown in figure 5 are called smectic walls. The energy associated with the case (a) (slope discontinuity) is :

$$
\sigma_{\mathrm{d}} \# K g\left(\theta_{\infty}\right) / d_{0}
$$

where $\theta_{\infty}$ is the angle between the layers and the $y$ axis. $g\left(\theta_{\infty}\right)$ increases with $\theta_{\infty}$ as $\theta_{\infty}^{3}$ for small $\theta_{\infty}$ (see below), and is of the order of 1 for $\theta_{\infty} \# \pi / 4$. Let us assume that the distance between walls is of the

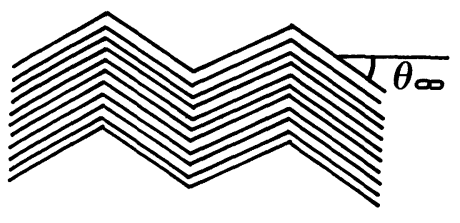

(a)

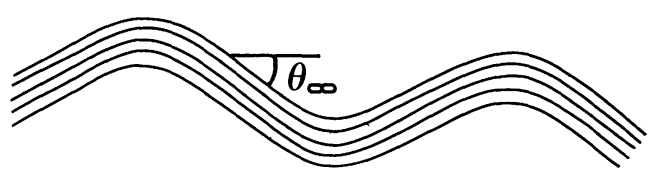

Fig. 5. - a) Discontinuity walls. b) curvature walls.

order $\Lambda=2 \pi / k_{\mathrm{c}}$ and that the angle $\theta_{\infty}$ is such that the extensional strain is relaxed : $\cos \theta_{\infty}=d_{0} / d$. With $X(H)=\left(d-d_{0}\right) / d$, this yields $\cos \theta_{\infty}=1-X$ the energy per unit surface is therefore :

$$
\begin{aligned}
F_{\mathrm{w}}=k_{\mathrm{c}} K g\left(\theta_{\infty}\right) / \lambda \sim & \\
& \sim K L_{x}^{-1 / 2} \lambda^{-3 / 2} \text { for } \theta_{\infty} \sim \pi / 4
\end{aligned}
$$

where $L_{x}$ is the width of the sample containing $\left\{L_{x} / d\right\}(+)$ and (-) layers. Expression (38) must be compared to the energy of the undulation solution. Of course, for large strain, expression (35) is no longer valid but one expects a form

$$
F_{\mathrm{s}} \sim K X^{2} / \lambda^{2}
$$

which is compatible with equation (36) for $X \gg X_{\mathrm{c}}$. Comparison of (38) and (39) shows that the discontinuity wall structure may be preferred for $X \geqslant\left(\lambda / L_{x}\right)^{1 / 4} \sim 0.3$ for the numerical values chosen above (this value corresponds to $d(H) \# 1.4 d_{0}$ i.e. to a field $H_{2} \sim 125 \mathrm{Oe}$. This may explain the observations of reference [1] that the sinusoidal profile transforms, for higher fields, to a wall structure.

Another possibility is that the walls are not characterized by a slope discontinuity but have a finite curvature.

The competition between the chevron structure with finite curvature at the tips and the undulating sine profile can be estimated by comparing the size $\Lambda$ of the curved tip (see Fig. $5 \mathrm{~b}$ ) with the period $\tilde{\Lambda}$ of the sine profile (31). Using the linear elasticity theory [8], $\tilde{\Lambda}$ is found to be of the order of $\tilde{\Lambda} \sim \lambda / \theta$. From the relation $\cos \theta=1-X$, one has $\theta \sim(2 X)^{1 / 2}$ which yields $\tilde{\Lambda} \sim \lambda /(2 X)^{1 / 2}$. Now, the chevron structure is preferred if $\tilde{\Lambda}<\Lambda\left(=\left(d L_{x}\right)^{1 / 2}\right)$ i.e. if $\lambda^{2} / 2 X<d L_{x}$ or using the definition of $\lambda(=\sqrt{K / B})$, $K / 2 B X<d L_{x}$. Therefore, for small $X$, the sine profile 
is preferred whereas for larger extensional constraint $X$, the chevron structure prevails. This discussion also illuminates the link between the narrowness of the tip $\tilde{\Lambda} \sim \lambda / \theta$ and the value of the curvature rigidity modulus $K$. For $\tilde{\Lambda}$ to be small, $\lambda$ and therefore $K$ must be small. In particular, $\tilde{\Lambda} \sim 0$ is possible only if $K \sim 0$.

Using the linear elasticity valid for small $\theta$, the surface free energy for the chevron structure with finite curvature at the tips is found to be [8] :

$$
\sigma_{\mathrm{d}} \#(2 / 3) K \theta^{3} / d_{0} \text {. }
$$

The corresponding energy per unit surface is :

$$
F_{\mathrm{w}}=k_{\mathrm{c}} K \theta^{3} / d_{0} \sim K L_{x}^{-1 / 2} \lambda^{-3 / 2} X^{3 / 2} .
$$

This is larger than expression (36) (which scales as $X^{2}$ for small $X$ ). This verifies that the undulation profile (31) is the preferred one and is the solution selected at the instability. The wall structure only appears at larger $X\left(X>X_{\mathrm{c}}\right)$. Note that the wall structure also admits the existence of « meta » dislocations responsible for a very rich topological structure [1-3].

At large $\theta(\theta>\pi / 4), \sigma_{d}$ increases rapidly. Indeed, the thickness of the walls scales as $d_{0} / \cos \theta$ and the corresponding energy is of the order of

$$
\begin{aligned}
\sigma_{\mathrm{d}}=\left(k_{\mathrm{c}} / d_{0}\right)\left(K / d_{0}^{2}\right)\left(d_{0} / \cos \theta\right. & \left.-d_{0}\right)^{2} \sim \\
& \sim k_{\mathrm{c}} K X^{2} / d_{0} .
\end{aligned}
$$

Note that this behaviour is different from the smectic case. Indeed, in the latter case, the discrete composition of the layers (molecules) insures that $\sigma_{\mathrm{d}}$ does not increase at large $\theta$ (in fact, $\sigma_{\mathrm{d}}$ decreases for $\theta>\pi / 4$ [8]).

4.2.2 « Fingering » instability. - At higher extensional strain (higher peak magnetic field), the discontinuity wall structure may destabilize into other structures whose topology is better tailored for relaxing the strain. A structure which seems to appear quite generally seems to be related (at least topologically) to a kind of fingering instability: a single domain of $(+)$ or (-) magnetization can destabilize from the spike of the chevron structure and grow in the $x$-direction as $H$ is decreased back to zero. This single process builds up a pair of disclinations of opposite signs forming a « transverse dislocation » (of Burgers vector $b$ ) and costs a rather large energy:

$$
F \sim K b / \lambda+(\pi K / 2) \log \left(b / 2 d_{0}\right)+\tau_{\mathrm{c}}
$$

$\tau_{c}$ is a constant term accounting for the inner core energy of the dislocation [8]. It is reasonable to expect that this structure starts from the spikes of the discontinuity walls with period $\sim k_{\mathrm{c}}^{-1}$. This would yield a 1Darray of alternate dislocations of global energy $F / b$ per unit length in the $y$-direction with $b \sim k_{\mathrm{c}}^{-1}$. The distorsion of neighbouring layers is small outside the disloca- tion array and the extensional strain can be relaxed with only a small additional cost (once the array has been formed) by increasing the length of the dislocations along $x$. Taking a structure with one dislocation per surface $b^{2}$ yields the energy of the structure :

$$
F_{\mathrm{T}} \sim\left\{K b / \lambda+(\pi K / 2) \log \left(b / 2 d_{0}\right)+\tau_{\mathrm{c}}\right\} b^{-2} .
$$

It becomes smaller than (42) when $(b \lambda)^{-1}<$ $L_{x}^{-1 / 2} \lambda^{-3 / 2} X^{2}$ i.e. $b>L_{x}^{1 / 2} \lambda^{1 / 2} X^{-2}$. Taking $b \sim \Lambda$ yields $X_{\min } \sim 1$ which is the order of magnitude of the minimal value of $X$ at which the discontinuity wall structure deforms into the "labyrinth » system. This means that these structures will occur near $X=1$ i.e. for very large $d(H)$ ( $H$ close to the saturation field). As these dislocations must be created randomly in the sample, when they grow, they interact and form complex random structures of interpenetrating dislocations.

\section{Conclusion.}

The main point of our analysis of the topological hysteresis observed in striped domain structures [1] is that these complex frustrated systems (characterized by long-range dipolar versus short-range exchange forces) can be described qualitatively and quantitatively by a smectic-like model relying on effective local interaction energies such as bending and compression energies.

It is proposed that this new language borrowed from the field of liquid crystals should be used more generally and could help developing new and powerful ways of reasoning for understanding the deformations and distortions in stripe magnetic structures.

If the present work permits us to rationalize some interesting experimental results [1] which had not been successfully explained up to now, it also opens many more questions. We now list some open questions for future work considered from the point of view of the liquid crystal analogy :

It is useful to distinguish between local features (points 1 and 2) and non-local features (point 3 to 8 ).

1) For high magnetic field peaks $(H \sim 180 \mathrm{Oe})$, local fingers grow out of the spikes of the discontinuity walls. This phenomenon appears to be isolated and not cooperative in its first stage. Is it a true instability (eigenvalue crossing the unit circle) ? It seems specific to the magnetic problem (magnetostriction ?). Therefore, what must be added to the smectic description to encapture this phenomenon?

2) Can one estimate the force exerted on a layer perpendicular to it and on the core of a (SDD) dislocation (parallel to the layer) by defects (inhomogeneities or plate thickness fluctuations) ? This aspect does not seem to have a smectic analogy and is therefore specific to the magnetic system. 
3) It is interesting to remark that the increase in topological complexity shown in figure 2 , as $H_{\max }$ increases, could be envisioned by a progressive decrease of the curvature elasticity modulus $K$ as defined in equation (14). Indeed, several facts enforce this view :

i) the decrease of $K$ as $H_{\max }$ increases is already encaptured in equation (14) (see $\S 3$ ) within the linear regime.

ii) the appearance of the discontinuity walls can be viewed as the signature of the crossing of the « instability » point $K=0$ (see the discussion of $\S 4.2 .1)$.

iii) the growth of the labyrinth structure is reminiscent of the class of systems which tend to maximize a functional of the curvature (maximize and not extremalize the curvature), subjected to some external 0 (and) internal constraints. This would occur in the smectic description as soon as $K$ becomes negative. This view will be explored in a forthcoming study.

One can develop an alternative view in which the complexity of the labyrinth structures, obtained at high maximum magnetic field, is related to the problem of filling a plane with constant thickness strips with a random population of defects. This is somewhat analogous to the construction of the focal conics in smectic liquid crystals. However, in the magnetic case, the presence of disorder seems to introduce a frustration. Therefore, the problem seems to be one of optimization (filling the plane with the minimum length of layers of constant width) in the presence of frustration.

Starting from $H_{\max }$ and decreasing $H$, the system evolves continuously from an unperturbed smectic to the labyrinth structure (see Fig. 2). During this process, there seems to be a ramification of structures as can be seen from the video recording of the experiments (Gouzerh, private communication). Is the notion of a common ancestor useful for these complex topologies? There seems to be an interesting analogy with the travelling salesman problem and therefore to spin glasses problems. If this is the case, then our local treatment would reach its limitation for describing the labyrinth structure and one should turn to other concepts.

4) The stripe structures are interesting systems for studying commensurate-incommensurate transitions. An external period could be imposed by a periodic modulation of the plate thickness. This would compete with the natural period $d_{0}$, and lead to a wealth of phenomena such as locking, incommensurate structures. This type of problem has interesting and important potential applications since it is considered for the conception of new magnetic memories [13]. Roughly, the idea would be to introduce line defects (vortices) in the Bloch walls: these Bloch line memories promise high storage densities up to $\sim 1.6 \mathrm{Gbit} / \mathrm{cm}^{2}$ for $0.5 \mu \mathrm{m}$ garnet films. The problem which has been considered consists in stabilizing the stripe domains with a periodic induced stress field at the garnet surface. Tensile (by evaporation of $\mathrm{Cr}$ bars) and compressive (by $\mathrm{Ne}^{+}$ implantation) stresses have been studied [14].

In the same vein, one can think of coupling two thin films of different thicknesses and therefore different equilibrium periods $\left(d_{1}\right.$ and $\left.d_{2}\right)$ by a small oxide layer. The modulated magnetic field induced by one film with period $d_{1}$ on the other will compete with the equilibrium period $d_{2}$. This leads to very rich behaviours such as commensurate, incommensurate, chaotic one-dimensional patterns, and will be studied in a forthcoming work.

5) What about the boundary effects ? Contrary to what happens in the smectic case, the layers appear to be able to join the borders at any angle indifferently with no cost in energy. This seems to be related to the absence of molecular structure in the 2D magnetic system compared to the usual liquid crystal for which the period of the structure is determined from the size of the building blocks.

6) We did not say anything about dynamics (motion of dislocation of fingers). In particular, the motion of the core of the dislocations is one-dimensional. Is it related to solitons?

7) How do the different instabilities (undulation, fingering) compete with the hierarchy of branching [5, 15] occurring as the plate thickness increases? This branching has no smectic analogy and brings a very rich zoology.

8) Finally, one could think of extending the validity of the smectic description to the upmost in order to describe the stripe $\rightarrow$ bubble transition. Qualitatively, one should introduce a topological term in the free energy accounting for the tendency for layers to break into pieces and form bubbles as one crosses the stripe $\rightarrow$ bubble transition.

These problems 1)-8), among others, which have been casted in the smectic language are interesting challenges for future studies.

\section{Acknowledgment.}

It is a pleasure to thank P. Coullet, F. Delyon, J. Gouzerh, J. C. Levy and J. L. Porteseil for useful remarks.

\section{Appendix.}

We now describe two factors which compete with the Peach-Koehler force discussed in paragraphe 3.2:

(i) the forces on dislocation core exerted by the magnetic field and by defects,

(ii) the attraction between dislocations of antiparallel Burgers vectors.

\section{A.1 EfFect of A MAGNetic FiEld on Dislocation CORES. - Due to the alternate $(+) /(-)$ layers structure,}


it is appropriate to distinguish between four different types of edge dislocations. As usual, the direction of the Burgers vector (parallel or antiparallel to the $x$ axis) distinguishes between left (right) semi-infinite edges. Furthermore, in each case, the edges can have either a positive or a negative magnetization. Therefore, we will use the notation $b_{+}(-)$for characterizing an edge dislocation of Burgers vector $b$ and edge magnetization $(+)\{(-)\}$.

Consider a dislocation $b_{-}$such as that depicted in figure 6. A net force along the dislocation in the $y$ direction results from the existence of a curvature at the core of the dislocation.

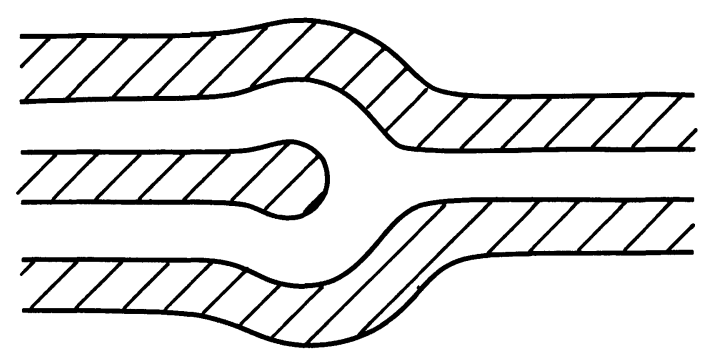

Fig. 6. - Schematic representation of a single dislocation. The dumb-bell form of the dislocation core stems from the favored state of the layer domain surrounded by an opposite magnetized domain.
In the proximity of the dislocation core of curvature $\sim 2 / d_{+(-)}$, the wall surface tension $r$ creates a Laplace pressure :

$$
p_{\mathrm{L}}=2 r / d_{+(-)} \text {. }
$$

It leads to a force excluding the $(+)$ or $(-)$ dislocations from the sample. Therefore, «stable " dislocations can only exist when the Laplace force is opposed by other pinning forces stemming for exemple from defects. We can think of different kinds of defects such as concentrations (leading to magnetization) inhomogeneities, or plate thicknesses fluctuations [14]...

In the presence of a magnetic field, $d_{(+)}\left(d_{(-)}\right)$ increases (decreases). As a consequence, for $b_{+(-)}$ dislocations, the Laplace pressure decreases (increases). For $b_{+}$dislocations, the excluding force diminishes with $H$. On the contrary, for $b_{-}$dislocations, the Laplace force increases with $H$ and tends to induce the motion of the $b_{-}$dislocations in the backward direction (leading therefore to the exclusion of the dislocations). Of course, the core may again be trapped by defects encountered on its route but eventually, as $H$ increases, $b_{-}$dislocations will tend to step out towards the borders of the sample. This Laplace term therefore reinforces the effect of the Peach-Koehler force.

More quantitatively, the Laplace force exerted on

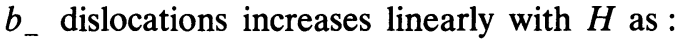

$$
\begin{aligned}
p_{\mathrm{L}} & =4 r / d+8 r / d^{2}\left(d / 2-d_{+(-)}\right) \\
& \sim 4 r / d+8 r h / \pi m_{\mathrm{s}} f_{0} d \\
& \sim 4 r / d+\left\{2 r g \mu_{\mathrm{B}} / \pi m_{\mathrm{s}} d\left(T-T_{0}\right)\right\}\left\{a T_{\mathrm{F}} / D\left(T_{q_{0}}-T\right)\right\} H
\end{aligned}
$$

The increase of $p_{\mathrm{L}}$ with $H$ (for $b_{-}$dislocations) given by equation (A.2) derives from the force exerted by the magnetic field on the walls parallel to the $y$-axis. It must be supplemented by the force exerted directly by the field on the parts of the walls parallel to the $x$-axis at the dislocations cores. Writing the magnetic interaction energy per unit volume as

$$
\mathrm{H}=-\mathbf{H} \cdot \mathbf{M}
$$

we obtain the additional pressure acting along a dislocation in the $y$-direction :

$$
p_{\mathrm{M}} \# d / 2 \int_{-\pi / 2}^{+\pi / 2}(\delta \mathbb{H} / \delta y) \cos \theta \mathrm{d} \theta
$$

where $\theta$ is the angle between the normal to the wall and the $y$-axis. $\delta M / \delta y$ is non-zero only in the vicinity of the core. For $b_{+}(-)$dislocations, we have approximately

$$
\delta M / \delta y \sim-(+) 2 m_{\mathrm{s}}\left(g y_{\mathrm{B}}\right) \cos \theta \cdot 1 / \xi .
$$

Inserting $\delta M / \delta y$ in (A4) yields

$$
p_{\mathrm{M}} \sim+(-)\left(-\pi d m_{\mathrm{s}} g \mu_{\mathrm{B}} / 2 \xi\right) H .
$$

The sign indicates that the field tends to pull in (out) $b_{+}\left(b_{-}\right)$dislocations. This contribution reinforces the effect described by equation (A2). We thus write in short the force induced on $b_{-}$dislocations by a magnetic field :

$$
f_{\mathrm{H}}=\left\{\left(p_{\mathrm{L}}-4 r / d\right)+p_{\mathrm{M}}\right\} D d=\gamma H
$$

$\gamma$ can be extracted from equation (A.2) and (A.5) :

$$
\gamma=\left\{2 r g \mu_{\mathrm{B}} / \pi m_{\mathrm{s}} d\left(T-T_{0}\right)\right\}\left\{a T_{\mathrm{F}} / D\left(T_{q_{0}}-T\right)\right\}+\pi d m_{\mathrm{s}} g \mu_{\mathrm{B}} / 2 \xi D d
$$

$\gamma$ is typically of the order of $\gamma \sim 10^{-6}-10^{-8}$ newtons oersted ${ }^{-1}$. Therefore, these effects cannot be neglected. 
A.1 Interaction BetWeEn Dislocations. - In most samples, dislocations seem to appear in pairs of opposite Burgers vectors [1]. These pairs are bounded by attractive forces mediated by the elastic deformation of the layers surrounding the dislocations.

The attraction between two dislocations can be calculated within the linear elasticity theory of smectic liquid crystals [8]. We just translate the results to our problem. The interaction energy for two edge-dislocations of Burgers vector $b_{1}$ and $b_{2}$ is given by:

$$
W_{1}=1 / 4 b_{1} b_{2} B\left(\lambda / \pi\left|x_{1}-x_{2}\right|\right)^{1 / 2} \exp \left\{-\left(y_{1}-y_{2}\right)^{2} / 2 \lambda\left|x_{1}-x_{2}\right|\right\}
$$

$\lambda$ is the penetration length $(\lambda=\sqrt{K / B})$.

The corresponding force obeys the relation :

$$
\mathbf{f}=-\nabla W_{1} .
$$

The component parallel to $y$ is therefore

$f_{y}=1 / 4 b_{1} b_{2} B\left(\lambda / \pi\left|x_{1}-x_{2}\right|\right)^{1 / 2}\left\{\left|y_{1}-y_{2}\right| / \lambda\left|x_{1}-x_{2}\right|\right\} \exp \left\{-\left(y_{1}-y_{2}\right)^{2} / 2 \lambda\left|x_{1}-x_{2}\right|\right\}$.

It is attractive (negative) for $b_{1} b_{2}<0$ and reaches its maximum for $\left|y_{1}-y_{2}\right|=\left\{\lambda\left|x_{1}-x_{2}\right|\right\}^{1 / 2}$ :

$$
\left|f_{y}^{\max }\right| \sim 0.09 b_{1} b_{2} B /\left|x_{1}-x_{2}\right|
$$

Noting $b_{1}=n_{1} d, b_{2}=n_{2} d$ and $\left|x_{1}-x_{2}\right|=n d$, where $n_{1}, n_{2}, n$ are integers, yields :

$$
\left|f_{y}^{\max }\right| \sim 0.09 B d n_{1} n_{2} / n \sim(10 K / d) n_{1} n_{2} / n .
$$

This shows that there exists a discrete hierarchy of barriers indexed by $n_{1} n_{2} / n$. When the pulling force exerted by the magnetic field increases, distant ( $n$ large) small Burgers vectors $\left(n_{1}, n_{2}\right.$ small) dislocations pairs are first extracted.

Note that the preceding results do not apply when $x_{1}=x_{2}$. In this case, the local disruption of the layers in the region between the cores are solution of :

$$
\lambda^{-2} \partial^{2} u / \partial x^{2}-\partial^{4} u / \partial y^{4}=0
$$

with the boundary conditions $u\left(x=0, y<y_{1}\right)=$ $\left(b_{1}-b_{2}\right) / 2, \quad u\left(x=0, y_{2}<y\right)=-\left(b_{1}-b_{2}\right) / 2$ and $u\left(x=0, y_{1}<y<y_{2}\right)=-\left(b_{1}+b_{2}\right) / 2$. This yields

$$
W_{1} \sim b_{1} b_{2} B \exp \left\{-\left(y_{1}-y_{2}\right)^{2} / 2 d^{2}\right\} .
$$

\section{Reference}

[1] Molho, P., Gouzerh, J., Levy, J. C. S. and Porteseil, J. L., J. Magn. Magn. Mat. 54-57 (1986) 857.

[2] Kooy, C. and Enz, U., Philips Res. Repts. 15 (1960) 7.

[3] see for example Malozemoff, A. P. and SLONCZWSKI, J. C., Magnetic domain walls in bubble materials (Academic Press, New York) 1979.

[4] Garel, T. and Doniach, S., Phys. Rev. B 26 (1982) 325.

[5] Gabay, M. and Garel, T., Phys. Rev. B 33 (1986) 6281.

[6] Grinstein, G. and Pelcovitz, R. A., Phys. Rev. Lett. 47 (1981) 856.

[7] Ribotta, R. and Durand, G., J. Physique 38 (1977) 179.

[8] Kleman, M., Points, lignes, parois, Tome 1 (Les Editions de Physique, CNRS) 1977.
[9] see for example Cellular structures in instabilities, Lect. Notes Phys. 210, ed. Wesfreid, J. E. and Zaleski, S. (Springer-Verlag) 1984.

[10] Coullet, P., Non-linear Phenomena in dissipative systems, Preprint 1986.

[11] Barker, W. A. and Gehring, G. A., J. Phys. C 19 (1986) 259.

[12] Kleman, M., Défauts étendus dans les smectiques in "Dislocations et déformations plastiques", Ecole d'été d'Yravals (1979), eds Groh, P., Kubin, L. P. and Martin, J. L.

[13] Konishi, S., IEEE Trans. Mag. Mag 19 (1983) 1238.

[14] Arnaud, L. and Bolleau, F., J. Physique Colloque 46 (1985) C6-137 and Klein, D. and Engemann, J., J. Appl. Phys. 57 (1985) 4071.

[15] SzymczaK, R., Piotrowski, K. and SzewczyK, A., Physica 113B (1982) 113, point 11.

[16] Mohlo, P. H., PhD Thesis, Grenoble (1985). 